\title{
Targeting translation: elF4E as an emerging anticancer drug target
}

\author{
CHUNWAN LU ${ }^{1,2}$, LEVI MAKALA ${ }^{3}$, DAQING WU ${ }^{3}$, YAFEI CAI ${ }^{1,2 *}$ \\ ${ }^{1}$ College of Life Sciences, Anhui Normal University, Key Laboratory of Biotic Environment and Ecological Safety \\ in Anhui Province, Wuhu 241000, Anhui, China, ${ }^{2}$ Department of Biochemistry and Molecular Biology, Medical \\ College of Georgia, Georgia Regents University, Augusta, GA 30912, USA, and ${ }^{3}$ Cancer Center, Medical College \\ of Georgia, Georgia Regents University, Augusta, GA 30912, USA
}

\begin{abstract}
The translation initiation factor eIF4E mediates a rate-limiting process that drives selective translation of many oncongenic proteins such as cyclin D1, survivin and VEGF, thereby contributing to tumour growth, metastasis and therapy resistance. As an essential regulatory hub in cancer signalling network, many oncogenic signalling pathways appear to converge on eIF4E. Therefore, targeting eIF4E-mediated cap-dependent translation is considered a promising anticancer strategy. This paper reviews the strategies that can be used to target eIF4E, highlighting agents that target eIF4E activity at each distinct level.
\end{abstract}

\section{Introduction}

Translation control, a tightly regulated process, plays a critical role in cell growth, proliferation and differentiation. Among the four consecutive stages of translation (initiation, elongation, termination and ribosome recycling), more attention was paid to initiation (Ref. 1). At this stage, the $43 \mathrm{~S}$ preinitiation ribosome complex is recruited to the $5^{\prime}$ terminus of mRNA through translation initiation complex eIF4F (Ref. 2). eIF4F is a heterotrimer complex binding the $5^{\prime}$-terminal cap structure 7-MeGpppN ( $\mathrm{N}$ is any nucleotide). eIF4F consists of eIF4E, the $5^{\prime}$ cap mRNA-binding protein; eIF4A, an ATP-dependent helicase unwinding the secondary structure of mRNA; and eIF4G, a scaffolding molecule serving a docking function in the assembly of eIF4F complex (Ref. 3). eIF4E mediates the association of eIF4F with cap structure and promotes recruitment of ribosome to the $5^{\prime}$ end of mRNA, playing a vital role in regulating global translation rates. The role of eIF4E in translation regulation unrelated to initiation, such as export of some specific mRNAs (e.g. cyclin D) from nucleus to cytoplasm (Refs 4,5$)$, has also been emphasised.

Cellular mRNAs can be categorised into two groups according to their structure property and inherent 'translatability': strong mRNAs (e.g. housekeeping genes), which have relatively short, unstructured $5^{\prime}$ UTRs (less $\mathrm{C}+\mathrm{G}$ content); and weak mRNAs, which have lengthy, highly structured $5^{\prime}$ UTRs $(\mathrm{G}+$ $\mathrm{C}$ rich) (Refs 6, 7). The significant difference between them is that weak mRNAs are much more dependent on eIF4E availability and poorly translated under normal conditions when eIF4F complex formation is limited. These mRNAs predominantly encode proteins including proto-oncogenes that regulate hallmark capabilities of cancer cells. When eIF4E is overexpressed or hyperactive, translation of weak mRNAs is selectively and disproportionately enhanced, while strong mRNAs are only minimally affected by alteration in eIF4F complex formation.

Several studies have demonstrated that elevated eIF4E levels preferentially increase mRNA translation involved in all aspects of malignancy, such as protooncoproteins (e.g. c-myc, cyclin D1, ODC, survivin), angiogenesis factors (e.g. FGF2 and VEGF) and degradative enzymes (e.g. MMP9) (Ref. 8). The list of mRNAs controlled by eIF4E is ever-increasing. Moreover, increased eIF4E up-regulates the nucleocytoplasmic transport of mRNAs encoding potent growth and survival proteins, such as cyclin D1 (Ref. 9). Therefore, eIF4E level affects transformation, tumourigenesis, metastasis, and drug resistance in both experimental cancer models and human cancer tissues. Indeed, its overexpression is common in multiple cancer types, including malignancies of prostate, breast, head and neck, stomach, colon, lung, skin, oesophagus, bladder, cervix and the hematopoietic system (Refs 10, 11). Also, elevated eIF4E levels may serve as a biomarker predicting disease progression, overall survival, or relapse after definitive therapy (Refs 12, 13). On the contrary, knockdown eIF4E by small interfering RNA (siRNA) can suppress oncogenic transformation (Refs 14, 15, 16).

The activation of eIF4E, which functions as a regulatory hub of many major oncogenic pathways, is a crucial event of the PI3K/AKT/mTOR pathway. Consequently, it has attracted considerable attention as a promising target for anticancer drug discovery in 
practice (Ref. 7). This review provides a comprehensive overview of strategies applicable for developing eIF4Etargeted agents.

\section{elF4E regulation and targeting strategies}

eIF4E is regulated at multiple levels, including gene expression, sequestration and phosphorylation, etc. (Refs 17).

At the transcriptional level, Myc is one of the best known transcription factors, which can activate eIF4E gene through two Myc-binding sites (E-boxes) in the eIF4E promoter (Ref. 7). At post-transcriptional level, HuR, a transcriptional factor, is responsible for stabilising eIF4E mRNA (Ref. 18). Post-translationally, eIF4E can be ubiquitinated primarily at Lys-159 and go through proteasome-dependent degradation (Ref. 19).

The interaction of eIF4E with eIF4 G is indispensible for cap-dependent translation initiation. A group of factors generally known as eIF4E inhibitory proteins modulate the eIF4E-eIF4G interaction (Ref. 20). The most well-studied eIF4E inhibitory proteins are 4EBPs, which sequester free-state eIF4E from eIF4G and block eIF4F complex formation. This sequestration results in the repression of translation of certain mRNAs that normally require high levels of available eIF4E (Ref. 20). Upon nutrients, energy, growth factors and stress stimulation, 4EBPs become phosphorylated at different sites as a consequence of the activation of PI3 K/AKT/mTOR signalling pathways. 4E-BP1 is one of the direct substrates of mTOR Complex 1 (mTORC1). Phosphorylated 4E-BP1 releases eIF4E, which is then free to associate with eIF4G to stimulate translation initiation (Refs 21, 22). Besides 4EBPs, the newly discovered eIF4E inhibitory proteins (e.g. Maskin and Cup) associate with eIF4E only on specific mRNAs through interactions with RNA-binding proteins (Ref. 20).

In addition, eIF4E itself has been shown to be phosphorylated in cancer cells, which is a prerequisite for the activity of eIF4E in cancer cells, whereas dispensable for normal development (Ref. 23). Thus, an increased level of phosphorylated eIF4E was found in a broad spectrum of cancer cell lines (Ref. 24). Phosphorylation of eIF4E (Ser209) is mediated by the MAP kinase-interacting protein kinases (Mnk1 and Mnk2), which are in turn activated by ERK and p38 MAPK pathways (Refs 25, 26).

Till now, eIF4E-targeted strategies should include: targeting eIF4E synthesis; targeting eIF4F complex integrity (antagonising eIF4E-to-cap and eIF4E-eIF4 $\mathrm{G}$ interaction); sequestration of eIF4E and phosphorylations of eIF4E. A summary of the strategies are shown in Figures 1 and 2. Therapeutic agents derived from these strategies that have been developed are summarised in Figure 3 and Table 1 and will be reviewed in detail.
Targeting elF4E synthesis: siRNA and antisense oligonucleotide (ASO)

eIF4E overexpression leads to the development of lymphomas and other cancers in vitro and in vivo (Ref. 99), directly targeting eIF4E mRNA by siRNA (Refs 14, $15,100,101)$ or specific oligonucleotide (ASO) (Refs $27,28,102,103$ ) has been extensively studied.

eIF4E knockdown by siRNA resulted in cell cycle arrest, suppression of colony formation, inhibition of cell mobility and enhanced chemosensitivity in MDAMB-231 triple negative (TN) breast cancer cells (Ref. 14). Although eIF4E knockdown inhibited growth in various breast cancer cell lines, it did not lead to activation of Akt (Ref. 15). In addition, eIF4E knockdown can suppress cell growth in endometrial adenocarcinoma and squamous carcinoma (Refs 100, 101). Tumour-specific RNAi via using survivin promoter-driven RNA interference system was recently demonstrated to reduce eIF4E gene expression effectively and specifically, resulting in apoptosis, growth inhibition and enhancement of chemosensitivity to cisplatin in breast cancer cells both in vitro and in vivo (Ref. 16). Moreover, eIF4E silencing enhanced radiosensitivity of tumour cells, while has no effect on normal cells (Ref. 104).

Antisense RNA (asRNA), a single-stranded RNA, can be introduced into cells to inhibit the translation of a complementary mRNA by base pairing to it and physically obstructing translation machinery (Ref. 105). Down-regulation of eIF4E via asRNA slowed down soft agar growth, increased tumour latency, and accelerated the rates of tumour-doubling times (Ref. 106). asRNA therapy for eIF4E can also be used as adjuvant therapy for head and neck cancers, particularly in cases in which elevated eIF4E is found in the surgical margins (Ref. 107).

ASOs are unmodified or chemically modified singlestranded DNA molecules. In general, they are relatively short (12-25 nucleotides) and hybridise to complementary target mRNAs by Watson-Crick base pairing (Ref. 108). ASOs have been used to selectively inhibit thousands of genes in mammalian cells and multiple genes in humans. There are over 20 antisense drugs currently in clinical trials, some of which are showing encouraging results (Ref. 109). First generation ASOs contained a phosphorothioate (a sulfur substitution of a non-bridging $\mathrm{O}$ ) backbone, whereas second generation ASOs, contained the phosphorothioate backbone plus $2^{\prime}$-O-methoxyethyl modification of riboses at the $5^{\prime}$ and $3^{\prime}$ ends. These modifications enhance affinity for targeted RNA, thus increasing stability and potency, improving antitumour potential and decreasing toxicity (Ref. 110). Similarly, eIF4E ASOs were designed specifically to recruit endogenous RNase $\mathrm{H}$ and decreased eIF4E expression at the mRNA level (Refs 111,112).

Graff and colleagues designed ASOs capable of targeting both murine and human eIF4E and evaluated their effects on eIF4E reduction in both human 


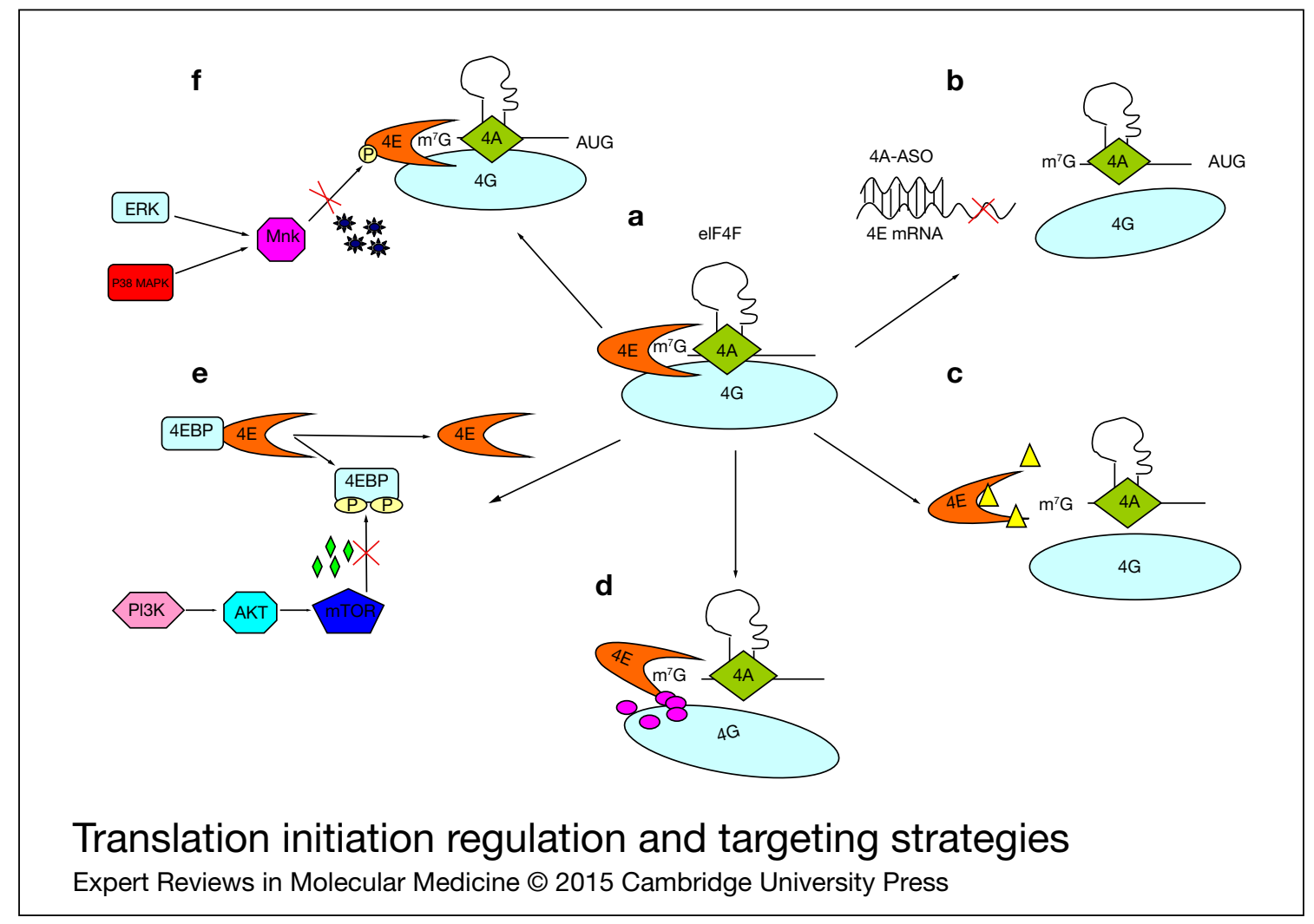

FIGURE 1 .

Translation initiation regulation and targeting strategies. (a) Translation initiation complex eIF4F is regulated at distinct levels. (b) siRNA or ASO degrade eIF4E mRNA to reduce its expression. (c) Ribavirin or nucleotide analogues disrupt interactions between eIF4E and Me ${ }^{7} \mathrm{G}-$ capped mRNA. (d) Small molecules disrupt eIF4E/eIF4 G association. (e) Memetic peptides or mTOR inhibitors sequester eIF4E. (f) Mnk inhibitors prevent eIF4E activation. 4E, eIF4E; 4G, eIF4G; 4A, eIF4A; 4EBP, eIF4E-binding protein; ASO, antisense oligonucleotide.

xenografts and normal mouse tissues (Ref. 27). These ASOs decreased eIF4E proteins and had dramatic cytotoxic effects at nanomolar concentrations across a panel of human cancer cell lines, including prostate cancer, breast cancer, head and neck cancer, non-small cell lung cancer and mesothelioma (Refs 27, 102, 103). These ASOs also repressed expression of eIF4E-regulated proteins, inducing apoptosis as well as preventing angiogenesis (Refs 27, 102, 103). Most importantly, intravenous ASO administration selectively and remarkably reduced eIF4E expression in human tumour xenografts, significantly suppressing tumour growth without obvious changes of body and organ weight, or liver transaminase levels (Ref. 27). A phase I dose escalation design was used to determine the dose level of eIF4E ASO LY2275796 that could be safely administered to patients with advanced solid tumours. LY2275796 was well tolerated at the $1000 \mathrm{mg}$ dose level with only mild toxicities (grades 1-2), which meant it has alluring prospect of clinical application (Ref. 28).

\section{Antagonising elF4E/cap interaction}

\section{Ribavirin}

Targeting the interaction of eIF4E and 7-MeG-Capped mRNA becomes attractive because an effective compound based on this target should inhibit eIF4E binding to capped mRNA specifically and block translation initiation subsequently. One typical approach to this is ribavirin (1- $\beta$-D-ribofuranosyl-1, 2, 4-triazole3 -caboxamide), which shares similar structure to 7-MeGTP (Refs 29, 113). It was observed that ribavirin bound to eIF4E with micromolar affinity and competed with eIF4E:mRNA binding (Ref. 30). At the same time, it impaired eIF4E-dependent Akt survival pathways and potently inhibited the biological effect of eIF4E (Ref. 31). As a result, it suppressed eIF4Emediated oncogenic transformation as well as tumour growth both in vitro and in vivo (Ref. 30).

The clinical efficacy of ribavirin in M4/M5 acute myeloid leukaemia (AML) patients was promising (Ref. 32) because it can cooperate with a wide variety of established agents to reduce the colony formation in primary AML specimens (Ref. 33). In a Phase II trial, ribavirin treatment benefits poor prognosis AML patients (Ref. 34). $10 \mathrm{mM}$ ribavirin was not cytotoxic to primary chronic lymphocytic leukaemia (CLL) lymphocytes in vitro, and significantly sensitised $76 \%$ of the samples tested with fludarabine (Ref. 35). Ribavirin also has effects on solid tumour and inhibits growth of several breast cancer cell lines with the elevated eIF4E level (Ref. 36). More interestingly, ribavirin effectively restored oestrogen receptor alpha (ESR1) gene expression alone and even more in 


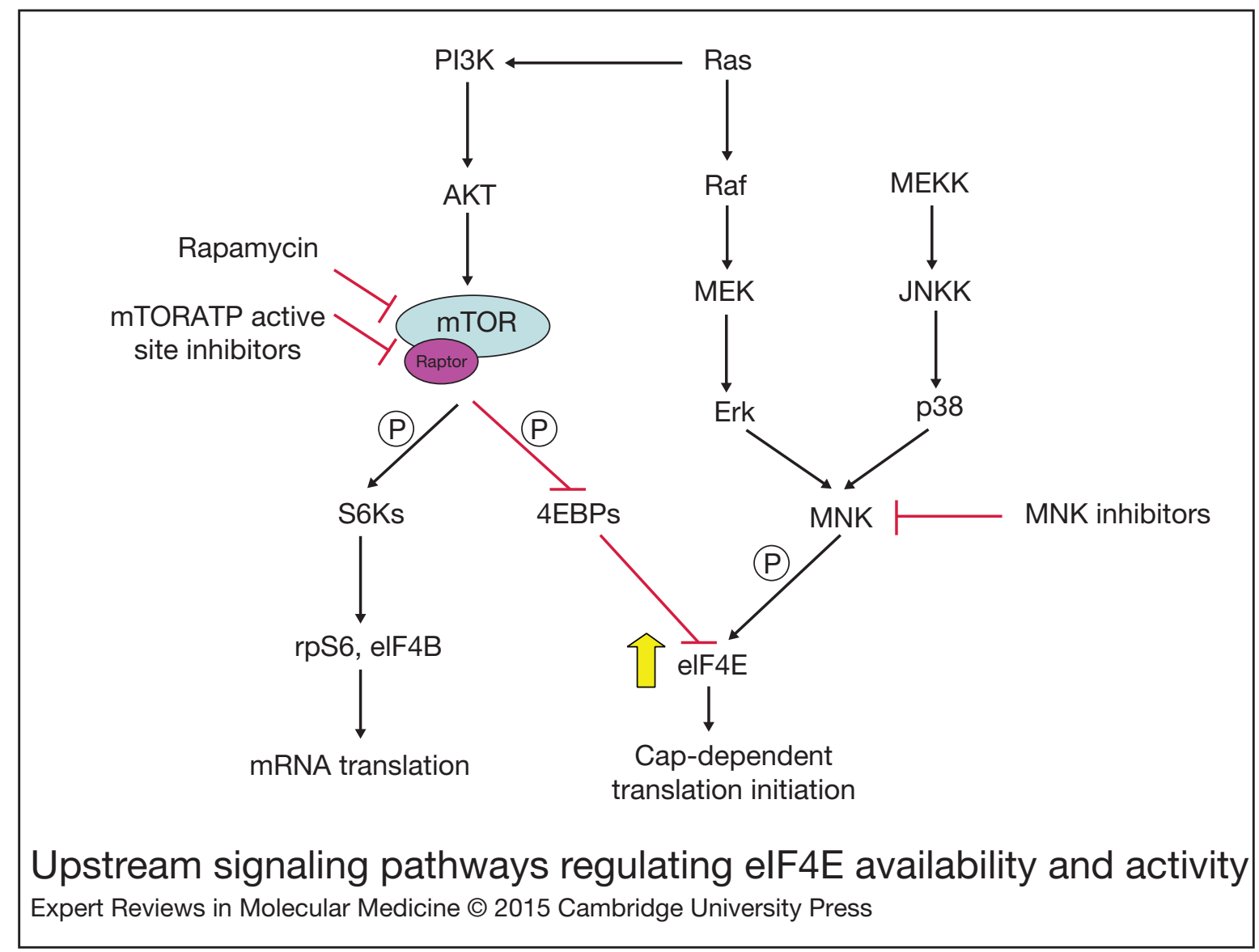

FIGURE 2.

Upstream signalling pathways regulating eIF4E availability and activity.

combination with suberoylanilide hydroxamic acid, leading to tamoxifen sensitivity restoration in ESR1 negative breast cancer cell lines (Ref. 37). Pharmacologic inhibition of eIF4E with ribavirin also improved tumour cell radiosensitivity (Ref. 104).

However, parallel studies have shown different outcomes. Yan and co-workers found that ribavirin was unable to function as a cap analogue in chemical cross-linking assays, cap-affinity chromatography and cap-dependent translation assays (Ref. 114). Independently, Westman et al. verified the findings of Yan et al. in a series of experiements (Ref. 115). In contrast, Kentsis et al. have rebutted their findings by suggesting specific binding of ribavirin to eIF4E through mass spectrometry detection (Ref. 116). These contrasting results may likely be because of different experimental methods and conditions.

\section{Other cap-binding antagonists}

Over the years, a large variety of nucleoside and nucleotide analogues derived from 7-MeGTP have been synthesised and evaluated for their ability of inhibiting eIF4E binding to capped mRNA specifically. Three independent groups (Refs 38, 117, 118) have developed libraries of 7-MeGMP analogues with favourable drug-like properties. Since aryl substitution at N7 has displayed a superior binding affinity
(Ref. 38), studies have therefore been focused on the utility of the synthetic nucleotide derivative 7-benzyl guanosine monophosphate (7-BnGMP) to block the binding of eIF4E to mRNA cap (Ref. 39). A recent crystallographic study with co-crystals of 7-BnGMP and eIF4E revealed that cap-binding pocket undergoes a unique structural change in order to accommodate the benzyl group (Ref. 40).

While effective in mammalian cell-free systems and zebrafish embryos, the efficacy of 7-BnGMP in cells is poor because of its low cell-membrane permeability. One approach to improve its in vivo activity is to develop a stable, cell-permeable prodrug (pro-nucleotide) which can be bio-activated within cells (Ref. 119). Phosphoramidates are promising prodrugs for this purpose in consideration of their low toxicity, high solubility and stability. In fact, there have been several successful examples of their applications for antiviral and anticancer therapies (Refs 120, 121).

Carston R. Wagner's group recently reported the synthesis of a novel class of Histidine Triad Nucleotide Binding Protein (HINT)-dependent pronucleotides that interdict epithelial-to-mesenchymal transition (EMT) (Ref. 41). 4Ei-1, one of the novel prodrugs, powerfully inhibited cap-dependent translation in zebrafish embryos without causing developmental abnormalities, and prevented eIF4E from triggering 


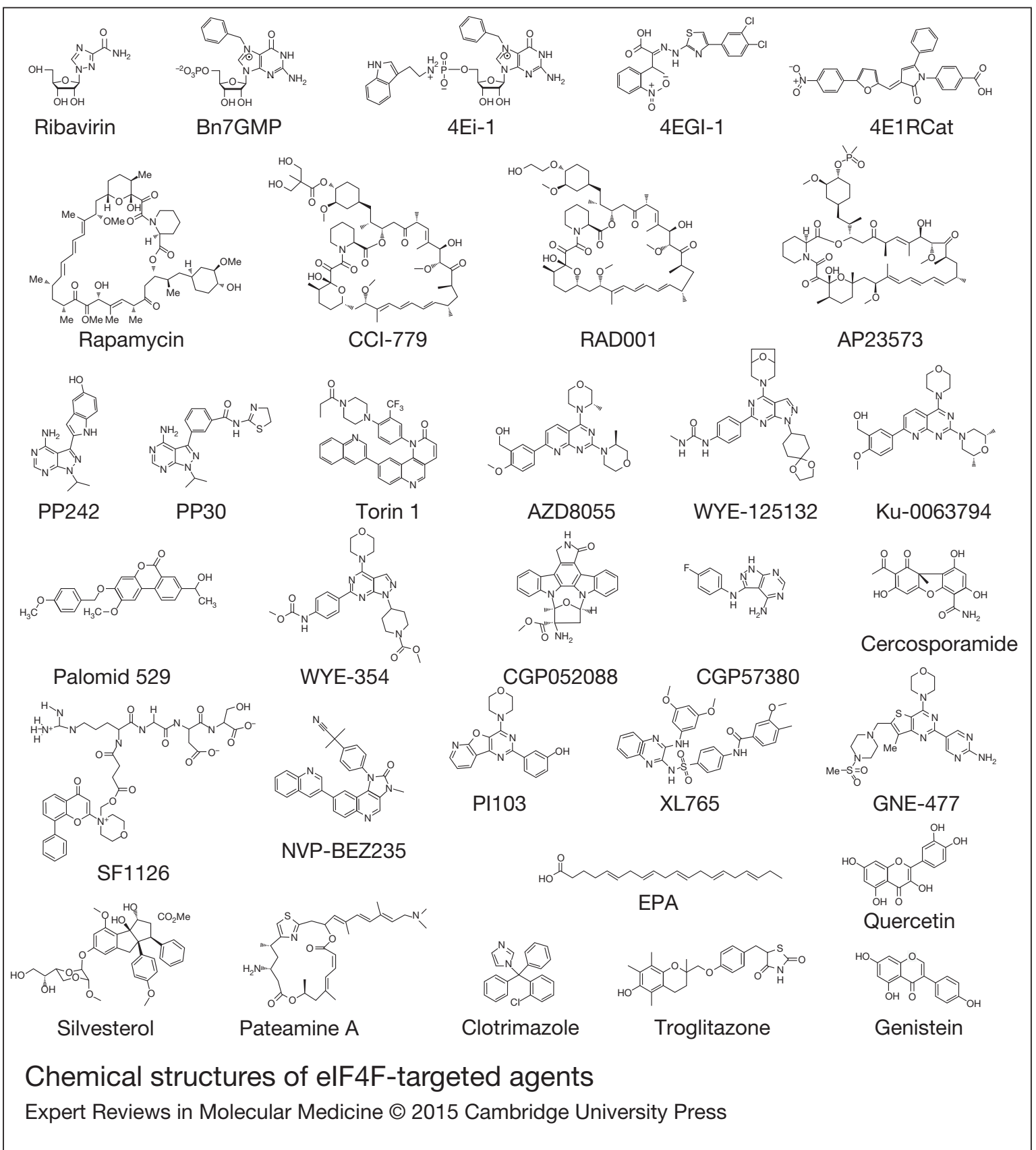

FIGURE 3.

Chemical structures of eIF4F-targeted agents.

EMT in zebrafish ectoderm explants without obvious toxicity (Ref. 41). Metabolism studies with whole cell lysates demonstrated that this prodrug was rapidly converted into active metabolite 7-BnGMP (Ref. 41). 4Ei-1 is the first nontoxic small molecule able to repress EMT by targeting the interaction of eIF4E with mRNA cap. More recently, Chen et al. pointed out that 4Ei-1 was a novel prodrug that reduced proliferation, suppressed colony formation, diminished association of eIF4E with the mRNA cap, and sensitised mesothelioma cells to pemetrexed (Ref. 42). Shui and co-workers showed that treatment of breast and lung cancer cells with 4Ei-1 resulted in chemosensitisation to gemcitabine and induced eIF4E proteasomal degradation, providing another mechanism of 4Ei-1 to induce translation inhibition except for down-regulation of eIF4E cap binding (Ref. 43).

\section{Targeting elF4E and elF4G interaction}

Studies have demonstrated that, eIF4E function is also regulated at the level of interactions with eIF4G and 4E-BPs (Ref. 20). This occurs on the dorsal surface of eIF4E, opposite to the cap-binding site. Binding of eIF4 G to eIF4E improves cap-dependent translation through recruitment of eIF4A and the eIF3-40S 
TABLE 1.

AGENTS TARGETING EIF4F AT DIFFERENT LEVELS

\begin{tabular}{|c|c|c|c|c|}
\hline Primary target & Agent & Agent class & Clinical stage & Reference \\
\hline eIF4E synthesis & eIF4E ASO LY2275796 & Oligonucleotide & Phase I & $(\operatorname{Refs} 27,28)$ \\
\hline \multirow[t]{3}{*}{ eIF4E-cap interaction } & Ribavirin & Nucleoside analogue & Phase II & (Refs $29,30,31,32,33,34,35,36,37)$ \\
\hline & $\mathrm{Bn}^{7} \mathrm{GMP}$ & Small organic & Laboratory & $(\operatorname{Refs} 38,39,40)$ \\
\hline & $4 \mathrm{Ei}-1$ & Small organic & Laboratory & (Refs $41,42,43)$ \\
\hline \multirow[t]{3}{*}{ eIF4E-eIF4G interaction } & 4EGI-1 & Small organic & Laboratory & (Refs $44,45,46)$ \\
\hline & 4E1RCat & Small organic & Laboratory & (Ref. 47) \\
\hline & $\begin{array}{l}\text { GnRH-4EBP fusion } \\
\text { peptide }\end{array}$ & Oligopeptide & Laboratory & (Ref. 48) \\
\hline eIF4E binding & 4EBP mimetic peptides & Oligopeptide & Laboratory & $(\operatorname{Refs} 49,50)$ \\
\hline \multirow[t]{4}{*}{ mTOC1 activity } & Rapamycin & Antibiotics & $\begin{array}{l}\text { FDA } \\
\quad \text { approved }\end{array}$ & (Refs $51,52,53,54,55,56,57)$ \\
\hline & CCI-779 & $\begin{array}{l}\text { Rapamycin } \\
\text { analogues }\end{array}$ & $\begin{array}{l}\text { FDA } \\
\text { approved }\end{array}$ & $($ Refs $58,59,60,61,62,63,64,65)$ \\
\hline & RAD001 & $\begin{array}{l}\text { Rapamycin } \\
\text { analogues }\end{array}$ & Phase II & (Ref. 66) \\
\hline & AP23573 & $\begin{array}{l}\text { Rapamycin } \\
\text { analogues }\end{array}$ & Phase II & (Ref. 67) \\
\hline \multirow{8}{*}{$\begin{array}{l}\text { mTOC } 1 \text { and mTOC2 } \\
\text { activity }\end{array}$} & PP242 & Small organic & Laboratory & (Ref. 68) \\
\hline & PP30 & Small organic & Laboratory & (Ref. 68) \\
\hline & Torin 1 & Small organic & Laboratory & (Ref. 69) \\
\hline & AZD8055 & Small organic & Phase I & (Refs $70,71,72)$ \\
\hline & WYE-125132 & Small organic & Laboratory & (Ref. 73) \\
\hline & Ku-0063794 & Small organic & Laboratory & (Ref. 74) \\
\hline & Palomid 529 & Small organic & Laboratory & (Ref. 75) \\
\hline & WYE-354 & Small organic & Laboratory & (Ref. 76) \\
\hline \multirow[t]{5}{*}{$\mathrm{P} 13 \mathrm{~K}$ and mTOR activity } & SF1126 & Small organic & Laboratory & (Ref. 77) \\
\hline & NVP-BEZ235 & Small organic & Phase I & (Refs 78, 79) \\
\hline & PI103 & Small organic & Laboratory & (Ref. 80) \\
\hline & XL765 & Small organic & Laboratory & (Ref. 76) \\
\hline & GNE-477 & Small organic & Laboratory & (Ref. 81) \\
\hline \multirow[t]{3}{*}{ Mnk activity } & CGP052088 & Small organic & Laboratory & (Ref. 82) \\
\hline & CGP57380 & Small organic & Laboratory & (Refs $83,84,85,86,87,88)$ \\
\hline & Cercosporamide & Small organic & Laboratory & $(\operatorname{Refs} 89,90,91)$ \\
\hline \multirow[t]{2}{*}{ eIF4A activity } & Silvesterol & Small organic & Laboratory & (Refs 92, 93) \\
\hline & Pateamine A & Small organic & Laboratory & (Ref. 94) \\
\hline \multirow[t]{5}{*}{ eIF2 activity } & Eicosapentaenoic Acid & Small organic & Laboratory & (Ref. 95) \\
\hline & Clotrimazole & Small organic & Laboratory & (Ref. 96) \\
\hline & Troglitazone & Small organic & Laboratory & (Ref. 97) \\
\hline & Genistein & Small organic & Laboratory & (Ref. 98) \\
\hline & Quercetin & Small organic & Laboratory & (Ref. 98) \\
\hline
\end{tabular}

ASO, antisense oligonucleotide; 4EBP, eIF4E-binding protein.

ribosomal subunit (Refs 17, 20). Thus, targeting the eIF4E/eIF4 G protein-protein interaction is a rational mechanism to repress cap-dependent translation.

\section{$4 E G I-1$}

The formation of eIF4E/eIF4 G complex is regulated by the 4E-BPs, which competes with eIF4G for binding to eIF4E (Ref. 122). Gerhard et al. developed a high-throughput fluorescence polarisation assay for identifying small-molecule inhibitors of eIF4E/eIF4 $\mathrm{G}$ interaction to pharmacologically mimic anti-eIF4F effect of 4E-BPs (Ref. 44). Among the 16000 compounds screened, the most potent one identified is 4EGI-1, which bound eIF4E, disrupted eIF4E/eIF4 $\mathrm{G}$ association, and inhibited cap-dependent translation. Interestingly, while 4EGI-1 displaced eIF4 G from eIF4E, it effectively enhanced 4EBP1 association both in vitro and in cells (Ref. 44). 4EGI-1 exhibits pro-apoptotic activity and represses the growth of multiple cancer cell lines. Its treatment caused cell death in
Jurkat cell lines and multiple myeloma cells, decreased proliferation of A549 lung cancer cell lines (Refs 44, 45). In primary AML cells, 4EGI-1 dramatically slowed down clonogenic growth of AML precursors and induced apoptosis in massive blast cells (Ref. 46), which thus represented an attractive option for the development of new therapies in AML. More recently, some researchers attempted to improve the physicochemical properties of 4EGI-1 to meet the urgent demand for clinical application (Refs 123, 124). Subsequently, a series of rigidified mimetic of 4EGI-1 were synthesised and characterised, which were more potent than the parent inhibitor (Ref. 125).

Although 4EGI-1 was discovered as a small molecule inhibitor that disturbed the interaction of eIF4E and eIF4G, Fan et al. revealed that 4EGI-1 sensitised human lung cancer cells by promoting TRAILmediated apoptosis (Refs 126, 127). In addition, cancer cells showed only 2-fold higher susceptibility to 4EGI-1 than their non-transformed counterparts. 
This inefficient therapeutic index, together with the significant 'off target' mechanism, dampens the original enthusiasm for this agent.

\section{E1RCat}

Another small molecule inhibitor 4E1RCat has been discovered (Ref. 47) after screening a library of 217 341 compounds via a time-resolved (TR)-fluorescence resonance energy transfer (FRET) method. 4E1RCat interfered with the interactions between eIF4E and eIF4 G or 4E-BP1. As a consequence, cap-dependent translation is suppressed. This compound can reverse tumour chemoresistance (doxorubicin) in $\mathrm{E} \mu-\mathrm{Myc}$ lymphoma mouse model by sensitising cells to the proapoptotic action of DNA damage (Ref. 47).

\section{Other agents targeting eIF4E and eIF4 G Interaction}

Cao et al. recently reported that Quabain, a kind of cardiac glycoside, directly bound eIF4E, destroyed eIF4E/eIF4 G association, thus inhibiting cap-dependent translation and down-regulating its critical target HIF-1. The association between Ouabain and eIF4E gave us a new clue of using cardiac glycosides for cancer therapeutics (Ref. 128). Perillyl alcohol, a kind of secondary product of plant mevalonate metabolism, also attenuated interactions between eIF4E and eIF4 G in prostate cancer cell lines (Ref. 129).

\section{Sequestering elf4E}

\section{$4 E B P$ mimetic peptides directly binding eIF4E}

As mentioned above, 4EBP sequesters eIF4E and consequently prevents cap-dependent translation initiation. Reasonably, 4EBP mimetic peptides which directly bind with eIF4E can reduce free eIF4E level, just as 4EBPs do.

Recently, a strategy developed by Naora and coworkers is to use 4EBP-based peptides to sequester eIF4E (Ref. 48). They designed a peptide containing residues 49-68 of 4EBP1, and fused it to an analogue of gonadotropin-releasing hormone $(\mathrm{GnRH})$. GnRH agonist-4EBP fusion peptide efficiently repressed the growth of GnRH receptor-expressing tumour cells, but not receptor negative cells, which is suitable for a targeted strategy. Therefore, GnRH-4EBP fusion peptide has the potential to treat ovarian cancer because this kind of cancer is hardly cured by conventional chemotherapies.

In addition, a eukaryotic expression vector $\mathrm{pSecX}-$ t4EBP1 was skilfully constructed, which contained phosphorylation defective 4E-BP1 domain and the protein transduction domain. The former domain down-regulated the expression of eIF4E by direct binding, and the latter domain can help plasmid penetrate the cellular membrane and enhance the efficiency of vector's spread. Interestingly enough, this plasmid significantly down-regulated tumour growth and improved the radiosensitivity of mouse breast carcinoma allografts in BALB/C mice model (Ref. 49). Moreover,
Terence et al. designed a series of peptides based on the conserved eIF4E-binding motifs linked to the penetratin peptide-carrier sequence. These peptides can effectively bind recombinant human eIF4E in vitro and induce apoptosis after introduction into MRC5 cells (Ref. 50).

\section{Rapamycin and Rapalogues}

In general, normalising deregulated eIF4F-mediated translation can be accomplished indirectly by interrupting upstream signals leading to eIF4E dissociation from 4EBPs. Naturally, targeting mTOR signalling pathway is a therapeutically attractive option for the purpose of sequestering eIF4E because 4EBPs are direct substrates of mTOR kinase (Ref. 130).

So far, rapamycin (sirolimus) and its analogues are the most well characterised mTOR inhibitors. As an immunosuppresive drug, rapamycin can also act as a cytostatic agent, preventing growth of various tumour cell lines including renal cancer, small cell lung cancer, and pancreatic cancer cells (Refs 51, 52, 53). One potential mechanism explaining its antiproliferative effects is the prevention of phosphorylation of 4E-BP1 by mTORC1, which in turn sequesters eIF4E and restrains the initiation of cap-dependent translation (Ref. 54).

Although rapamycin has shown promising antitumour effects in several experimental tumour models, its clinical trials as an anticancer drug is unsuccessful at present. Therefore, several rapamycin analogues (rapalogues) with more favourable pharmaceutical characteristics have been developed, such as CCI-779 (Temsirolimus), RAD001 (Everolimus), AP23573 (Deforolimus,), SAR943 (32-deoxorapamycin) (Ref. 131) and ABT578 (zotarolimus) (Ref. 132). CCI-779, RAD001 and AP23573, which are currently under clinical trials, have shown obvious antitumour effects against a diverse range of cancer types in preclinical studies. CCI-779 inhibited mTOR function in several breast cancer cell lines (Ref. 58). Antiangiogenic effects may substantially contribute to its antitumour activity in breast cancer (Ref. 59). Also, CCI-779 can potentially be used as an adjuvant therapy in head and neck squamous cell cancer (Ref. 60), which has showed remarkable efficacy in PTEN-deficient tumours (Ref. 61). As such, CCI-779 recently became the first FDA-approved mTOR-targeted agent based on a phase III trial in advanced renal cell carcinoma patients (Ref. 62). In haematologic malignancies, CCI-779 has substantial single-agent activity in relapsed mantle cell lymphoma (MCL) patients (Ref. 63). RAD001, the only orally active rapamycin derivative, possessed antitumour activity in MCL and diffuse large B-cell lymphoma (Ref. 66). A phase II clinical trial demonstrated that AP23573 was well tolerated and had antitumour activity in patients with heavily relapsed or refractory hematologic malignancies (Ref. 67).

Rapamycin and rapalogues can also be used in combination with other chemotherapeutics. Simultaneous administration of rapamycin and EKI-785 (EGFR 
inhibitor) lead to synergistic antitumour effects in glioblastoma (GBM) multiforme cells (Ref. 55). Additionally, rapamycin increased the ability of cisplatin to induce apoptosis in human promyelocytic leukaemia cell line HL-60 and human ovarian cancer cell line SKOV3 (Ref. 56). Also, it can effectively restore tamoxifen response in breast cancer cells with aberrant Akt activity (Ref. 57). CCI-779 was able to restore cisplatin sensitivity in small cell lung cancer cell lines selected for cisplatin resistance (Ref. 64). Combination use of CCI-779 and gemcitabine achieved a significantly better survival in xenograft models of human pancreatic cancer compared with CCI-779 or gemcitabine alone (Ref. 65).

\section{Second-generation mTOR inhibitors}

Extensive research on mTOR has uncovered a complex network of regulatory loops that impact on mTOR-targeting approaches (Ref. 133) and may explain the inherent limitations of rapamycin-based strategies. For example, when mTORC1 is inhibited and unable to activate S6K, S6K-mediated feedback loop can lead to an up-regulation of PI3K signalling, and provide pro-survival and proliferative signals through Akt (Ref. 134). These loops, at least in some degree, counteract the effects of rapamycin in experimental cancer models and in patients (Ref. 133). Moreover, because mTORC 2 also plays a vital role in tumourigenesis (Ref. 135), the high selectivity of rapamycin for mTORC1 (Refs 136, 137) really triggers a major concern. Thirdly, rapamycin does not inhibit 4EBP phosphorylation by mTOR in some cells. One explanation of this is that it works through sterically blocking mTOR access to substrates, which is inefficient for a small substrate like 4EBP, while very efficient for large ones like S6K1(Refs 138, 139, 140). All the drawbacks mentioned above indicate that there exists an urgent need to search for second-generation mTOR inhibitors, which can sequester eIF4E more efficiently.

The second-generation inhibitors, which bind to the catalytic sites of mTOR, inhibit kinase activities of both mTORC1 and mTORC2 (Refs 68, 69, 70, 73, 74, 75,76 ). The active-site inhibitors of mTOR, PP242 and PP30, suppressed proliferation of primary cells more potently than rapamycin (Ref. 68). Torin1, another highly potent and selective ATP-competitive mTOR inhibitor, impaired cell growth and proliferation to a far greater extent than rapamycin (Ref. 69). AZD8055 (Refs 70, 71) and WYE-125132 (Ref. 73) are both ATP-competitive mTOR inhibitors and have antitumour activity in vitro and in vivo. Combination use of MEK1/2 inhibitor AZD6244 with AZD8055 enhanced the antitumour efficacy relative to the respective monotherapies in nude mouse xenograft models of human lung adenocarcinoma and colorectal carcinoma (Ref. 72). Ku-0063794 is a cell permeable and specific mTOR inhibitor, which inhibited mTOC1 and mTOC2 with an IC50 $10 \mathrm{nM}$ (Ref. 74). Palomid 529 reduced not only tumour growth, but also tumour angiogenesis and vascular permeability (Ref. 75). WYE-354, a novel pyrazolopyrimidine, displayed robust antitumour activity in PTEN null tumours (Ref. 76). All the above inhibitors can effectively minimise the feedback activation of Akt by mTORC2 to avoid offsetting their effects of sequestering eIF4E. More importantly, they more potently inhibit 4EBP phosphorylation compared with rapamycin and rapalogues.

Another class of small molecules related to mTOR kinase inhibitors is the mTOR and PI3 $\mathrm{K}$ dual specificity inhibitors. These molecules, such as SF1126 (Ref. 77), NVP-BEZ235 (Refs 78, 79), PI103 (Ref. 80), XL765 (Ref. 76) and GNE-477 (Ref. 81), simultaneously target ATP binding sites of mTORC and PI3 K with similar potency. NVP-BEZ235 and XL765 are undergoing clinical phase I trials. These inhibitors have the unique advantages to avoid PI3 K pathway reactivation caused by mTOR-p70S6 K negative feedback loop, so that they can exert their effects more thoroughly derived from sequestering eIF4E.

\section{Targeting elF4E phosphorylation}

\section{Role of Mnks in tumourigenesis and its regulation}

Although some success has been achieved on the inhibition of PI3 K/mTOR axis, the multiple feedback loops make this pathway disappointing, to some degree. The ideal goal should be to down-regulate the function of specific pathway in cancer cells without affecting normal cells and eliciting feedback loops that could impair the therapeutic efficacy. Phosphorylation of eIF4E by Mnks on Ser209 is critical for oncogenic activity of eIF4E (Refs 141, 142) Therefore, eIF4E phosphorylation has been established as a crucial event in tumourigenesis (Refs 26, 143), such as prostate cancer progression (Ref. 143). Reasonably, targeting Mnks could be the attractive therapeutic approach that in mammalian cells, Ser209 phosphorylation is not essential for the activity of eIF4E in normal cells but is required in cancer cells (Ref. 23). Takeshi and co-workers confirmed this viewpoint by demonstrating that Mnk1 and Mnk2 double knockout dramatically delayed the tumour development.

Mnk-eIF4 G interaction plays an essential role in the eIF4E phosphorylation regulation. Mnk interacts with the scaffolding protein eIF4G, which also binds eIF4E and brings Mnk and its substrate into physical proximity (Refs 144). From the aspect of negative regulation, Mnks can be dephosphorylated and deactivated by protein phosphatase 2A (PP2A), preventing further eIF4E phosphorylation (Ref. 144).

\section{Combination of targeting Mnk-eIF4E and mTOR pathways}

Mnk-induced eIF4E phosphorylation is closely related to mTOR pathway inhibition (Refs 145,146$)$. Two research groups have proposed a new strategy of 
enhancing mTOR-targeted cancer therapy through the combined treatment with Mnk inhibitors. Michal Marzec and co-workers reported that simultaneous inhibition of mTORC1 and Mnk markedly induced apoptosis of cutaneous $\mathrm{T}$ cell lymphoma cells (Ref. 147). The combined treatment also arrested the cell cycle progression and suppressed the growth of prostate cancer cells (Ref. 148). Furthermore, co-exposure to MNK1 and mTORC1 inhibitors profoundly decreased 4EBP1 phosphorylation, protein synthesis and proliferation in glioma cells, and reduced tumour growth in an orthotopic GBM mouse model (Ref. 149).

\section{Mnk inhibitors}

So far, three well-studied Mnk inhibitors have been reported: CGP052088, CGP57380 and Cercosporamide.

CGP052088 is a derivative of staurosporine, a broadspectrum kinase inhibitor. It inhibited Mnk1 with an IC50 value of $70 \mathrm{nM}$ in biochemical assays and was cytotoxic with a GI50 value of $4.5 \mu \mathrm{M}$ in a $24 \mathrm{~h}$ MTT proliferation assay (Ref. 82). CGP57380 was also found to be a potent Mnk1 and Mnk2 inhibitor in vitro (Refs 83, 84). Its antiproliferative effects in five breast cancer cell lines were primarily cytostatic, rather than cytotoxic, and were potentially because of the inhibition of cyclin D1 synthesis (Ref. 85). In addition, CGP57380 is therapeutically useful in blast crisis CML (Ref. 86). It enhanced imatinib activity against CML and overcame imatinib resistance through impairing polysome assembly (Ref. 87). Similarly, treatment of pancreatic cancer cell lines MiaPaCa2 and PT45P1 with CGP57380 in combination with gemcitabine caused a greater apoptotic cell death when compared with the use of either CGP57380 or gemcitabine alone (Ref. 88). Another Mnk inhibitor is Cercosporamide, an effective antifungal agent and phytotoxin extracted from the fungus Cercosporidium henningsii (Ref. 89), which suppressed AML precursors and enhanced the antileukaemic properties of cytarabine or mTOC1 inhibitors (Ref. 90). Furthermore, it can significantly down-regulate outgrowth of experimental B16 melanoma pulmonary metastases and subcutaneous HCT116 colon carcinoma xenograft tumours, without side effect on body weight. These findings confirmed that Mnk inhibition may provide a tractable cancer therapeutic approach (Ref. 91).

More recently, a novel Mnk inhibitor retinamides (RR), one of retinoic acid metabolism blocking agents, blocked eIF4E phosphorylation and subsequently restrained cell growth, colonisation, invasion, and migration, as well as induced apoptosis in TN and Her-2 overexpressing breast cancer cells through degrading Mnks rather than inhibiting its kinase activity just like the three Mnk inhibitors mentioned above (Ref. 150). Further, a series of 5-(2-(phenylamino pyrimidin-4-yl) tiazole-2 $(3 \mathrm{H})$-one derivatives have been discovered as selective Mnk2 inhibitors. They facilitated apoptosis in MV4-11 AML cells by reducing the expression of an antiapoptotic protein Mcl-1 (Ref. 151).

\section{Conclusions and outlook}

Our understanding of human cancer as a multi-factornetwork disease has led to the development of nextgeneration therapeutics. It is clear now that targeting regulatory hubs in the cancer signalling network instead of targeting individual genetic alterations will be more effective in treating a very heterogenous tumour. Accumulating evidences indicate that one of such hubs is eIF4E, serving as a node on which multiple oncogenic signalling pathways converge. As a result, eIF4E and translation initiation provide a promising target for cancer therapeutics. Indeed, enthusiasm for developing small molecule inhibitors blocking eIF4E function has lasted over the years.

In addition to the therapeutic strategies targeting eIF4E aforementioned, some other components of eIF4F complex should be considered as appealing oncology drug targets. These include eIF4A, an RNA helicase, which is frequently activated in cancer cells, either by its overexpression or by repression of the tumour suppressor Pdcd4 (programmed cell death 4) (Ref. 152). Silvesterol and Pateamine A are both eIF4A inhibitors. Silvesterol can effectively modulate the activity of eIF4A and repress translation initiation, exhibiting powerful anticancer activity in human breast cancer and prostate cancer xenograft models by inducing apoptosis and inhibiting angiogenesis (Refs 92, 93). In addition, the suppressive effects of Pateamine A (PatA) on translation are mediated through increasing the RNA-binding affinity of free eIF4A, thus sequestering eIF4A from the $4 \mathrm{~F}$ complex, which may lead to stalling of initiation complexes (Ref. 94).

The binding of initiator tRNA to the 40S ribosomal unit is mediated by translation initiation factor 2 (eIF2). Phosphorylation of $\alpha$-subunit of eIF2 prevents formation of the eIF2/GTP/Met-tRNA complex and stops global protein synthesis (Ref. 153). As a consequence, eIF2 is also a promising drug target at the level of translation initiation. eIF2 $\alpha$ can be phosphorylated by haeme-regulated inhibitor, PERK/PEK, and the double-stranded RNA-activated protein kinase (PKR) (Ref. 154). So far, reported eIF2 inhibitors include eicosapentaenoic acid (EPA) (Ref. 95), clotrimazole (Ref. 96), troglitazone (Ref. 97) and flavonoids (e.g. Genistein and Quercetin) (Ref. 98).

Despite the encouraging targeting potential of capdependent translation, our understanding of the role of eIF4E and other members regulating translation initiation in tumourigenesis remains rudimentary. For example, eIF4E is responsible for the regulation of multiple mRNAs involved in cancer progression; however, it is unclear if any of these eIF4E targets is indispensable. A comprehensive mapping of eIF4E target mRNAs will be imperative to elucidate the translational signature of eIF4E and its significance in 
human cancer. On the other hand, in order to improve the anticancer efficacy, future studies are needed to address the combination usage of eIF4E inhibitors with inhibitors targeting other oncogenic signalling pathways.

\section{Acknowledgement}

We thank Dr. Chunhong Yan for critical reading of this paper.

\section{Financial Support}

This study was supported by the National Natural Science Foundation of China (grant numbers 31372207 and 31572265); the National Supporting Projects for Science and Techniques (grant numbers 2012BAD12B10 and 2012BAD12B1003); and the Anhui Natural Science and Technology Program of Anhui Province (grant numbers 1208085MC41 and KJ2013A310).

\section{Conflict of Interest}

None.

\section{Ethical Standards}

I assert that all procedures contributing to this work comply with the ethical standards of NIH guidelines (NIH Pub. No. 85-23, revised 1996) and approved by Animal Care and Use Committee of Anhui Normal University. Approval number is '\#20150131'.

\section{References}

1. Sonenberg N. and Hinnebusch A.G. (2009) Regulation of translation initiation in eukaryotes: mechanisms and biological targets. Cell 136, 731-745

2. Fischer P.M. (2009) Cap in hand: targeting eIF4E. Cell Cycle 8, 2535-2541

3. Hsieh A.C. and Ruggero D. (2010) Targeting eukaryotic translation initiation factor 4E (eIF4E) in cancer. Clinical Cancer Research 16, 4914-4920

4. von der Haar T., Gross J.D., Wagner G. and McCarthy J.E. (2004) The mRNA cap-binding protein eIF4E in post-transcriptional gene expression. Nature Structural \& Molecular Biology 11, 503-511

5. Topisirovic I. et al. (2009) Molecular dissection of the eukaryotic initiation factor $4 \mathrm{E}$ (eIF4E) export-competent RNP. The EMBO Journal 28, 1087-1098

6. Jia Y., Polunovsky V., Bitterman P.B. and Wagner C.R. (2012) Cap-dependent translation initiation factor eIF4E: an emerging anticancer drug target. Medicinal Research Reviews 32, 786-814

7. Bitterman P.B. and Polunovsky V.A. (2012) Attacking a nexus of the oncogenic circuitry by reversing aberrant eIF4Fmediated translation. Molecular Cancer Therapeutics 11, $1051-1061$

8. De Benedetti A. and Graff J.R. (2004) eIF-4E expression and its role in malignancies and metastases. Oncogene 23, 31893199

9. Rousseau D., Kaspar R., Rosenwald I., Gehrke L. and Sonenberg N. (1996) Translation initiation of ornithine decarboxylase and nucleocytoplasmic transport of cyclin D1 mRNA are increased in cells overexpressing eukaryotic initiation factor 4E. Proceedings of the National Academy of Sciences of the United States of America 93, 1065-1070

10. Mamane Y. et al. (2004) eIF4E--from translation to transformation. Oncogene 23, 3172-3179

11. Konicek B.W., Dumstorf C.A. and Graff J.R. (2008) Targeting the eIF4F translation initiation complex for cancer therapy. Cell Cycle 7, 2466-2471
12. Flowers A. et al. (2009) Eukaryotic initiation factor 4E overexpression in triple-negative breast cancer predicts a worse outcome. Surgery 146, 220-226

13. Hiller D.J. et al. (2009) Predictive value of eIF4E reduction after neoadjuvant therapy in breast cancer. Journal of Surgical Research 156, 265-269

14. Zhou F.F. et al. (2011) Knockdown of eIF4E suppresses cell growth and migration, enhances chemosensitivity and correlates with increase in $\mathrm{Bax} / \mathrm{Bcl}-2$ ratio in triple-negative breast cancer cells. Medical Oncology 28, 1302-1307

15. Soni A. et al. (2008) eIF4E knockdown decreases breast cancer cell growth without activating Akt signaling. Molecular Cancer Therapeutics 7, 1782-1788

16. Dong K. et al. (2009) Tumor-specific RNAi targeting eIF4E suppresses tumor growth, induces apoptosis and enhances cisplatin cytotoxicity in human breast carcinoma cells. Breast Cancer Research and Treatment 113, 443-456

17. Martineau Y., Azar R., Bousquet C. and Pyronnet S. (2013) Anti-oncogenic potential of the eIF4E-binding proteins. Oncogene 32, 671-677

18. Topisirovic I. et al. (2009) Stability of eukaryotic translation initiation factor 4E mRNA is regulated by $\mathrm{HuR}$, and this activity is dysregulated in cancer. Molecular and Cellular Biology 29, 1152-1162

19. Murata T. and Shimotohno K. (2006) Ubiquitination and proteasome-dependent degradation of human eukaryotic translation initiation factor 4E. Journal of Biological Chemistry 281, 20788-20800

20. Richter J.D. and Sonenberg N. (2005) Regulation of capdependent translation by eIF4E inhibitory proteins. Nature 433, $477-480$

21. Tee A.R. and Blenis J. (2005) mTOR, translational control and human disease. Seminars in Cell \& Developmental Biology 16, 29-37

22. Wullschleger S., Loewith R. and Hall M.N. (2006) TOR signaling in growth and metabolism. Cell 124, 471-484

23. Hay N. (2010) Mnk earmarks eIF4E for cancer therapy. Proceedings of the National Academy of Sciences of the United States of America 107, 13975-13976

24. Fan S. et al. (2009) Phosphorylated eukaryotic translation initiation factor 4 (eIF4E) is elevated in human cancer tissues. Cancer Biology \& Therapy 8, 1463-1469

25. Hou J., Lam F., Proud C. and Wang S. (2012) Targeting Mnks for cancer therapy. Oncotarget 3, 118-131

26. Diab S. et al. (2014) MAP kinase-interacting kinases-emerging targets against cancer. Chemistry \& Biology 21, 441-452

27. Graff J.R. et al. (2007) Therapeutic suppression of translation initiation factor eIF4E expression reduces tumor growth without toxicity. Journal of Clinical Investigation 117, 2638-2648

28. Hong D.S. et al. (2011) A phase 1 dose escalation, pharmacokinetic, and pharmacodynamic evaluation of eIF-4E antisense oligonucleotide LY2275796 in patients with advanced cancer. Clinical Cancer Research 17, 6582-6591

29. Hofmann W.P., Herrmann E., Sarrazin C. and Zeuzem S. (2008) Ribavirin mode of action in chronic hepatitis C: from clinical use back to molecular mechanisms. Liver International: Official Journal of the International Association for the Study of the Liver 28, 1332-1343

30. Kentsis A., Topisirovic I., Culjkovic B., Shao L. and Borden K.L. (2004) Ribavirin suppresses eIF4E-mediated oncogenic transformation by physical mimicry of the 7-methyl guanosine mRNA cap. Proceedings of the National Academy of Sciences of the United States of America 101, 18105-18110

31. Tan K., Culjkovic B., Amri A. and Borden K.L. (2008) Ribavirin targets eIF4E dependent Akt survival signaling. Biochemical and Biophysical Research Communications 375, 341-345

32. Assouline S. et al. (2009) Molecular targeting of the oncogene eIF4E in acute myeloid leukemia (AML), a proof-of-principle clinical trial with ribavirin. Blood 114, 257-260

33. Kraljacic B.C., Arguello M., Amri A., Cormack G. and Borden K. (2011) Inhibition of eIF4E with ribavirin cooperates with common chemotherapies in primary acute myeloid leukemia specimens. Leukemia 25, 1197-1200

34. Borden K.L. and Culjkovic-Kraljacic B. (2010) Ribavirin as an anti-cancer therapy: acute myeloid leukemia and beyond? Leukemia \& Lymphoma 51, 1805-1815 
35. Martinez-Marignac V. et al. (2013) Pharmacological targeting of eIF4E in primary CLL lymphocytes. Blood Cancer Journal 3, e146

36. Pettersson F. et al. (2011) Ribavirin treatment effects on breast cancers overexpressing eIF4E, a biomarker with prognostic specificity for luminal B-type breast cancer. Clinical Cancer Research 17, 2874-2884

37. Sappok A. and Mahlknecht U. (2011) Ribavirin restores ESR1 gene expression and tamoxifen sensitivity in ESR1 negative breast cancer cell lines. Clinical Epigenetics 3, 8

38. Cai A. et al. (1999) Quantitative assessment of mRNA cap analogues as inhibitors of in vitro translation. Biochemistry 38, $8538-8547$

39. Jia Y. et al. (2010) Design, synthesis and evaluation of analogs of initiation factor 4E (eIF4E) cap-binding antagonist Bn7GMP. European Journal of Medicinal Chemistry 45, 1304-1313

40. Brown C.J., McNae I., Fischer P.M. and Walkinshaw M.D. (2007) Crystallographic and mass spectrometric characterisation of eIF4E with N7-alkylated cap derivatives. Journal of Molecular Biology 372, 7-15

41. Ghosh B. et al. (2009) Nontoxic chemical interdiction of the epithelial-to-mesenchymal transition by targeting cap-dependent translation. ACS Chemical Biology 4, 367-377

42. Chen E.Z. et al. (2014) Small-molecule inhibition of oncogenic eukaryotic protein translation in mesothelioma cells Investigational New Drugs 32, 598-603

43. Li S. et al. (2013) Treatment of breast and lung cancer cells with a N-7 benzyl guanosine monophosphate tryptamine phosphoramidate pronucleotide (4Ei-1) results in chemosensitization to gemcitabine and induced eIF4E proteasomal degradation. Molecular Pharmaceutics 10, 523-531

44. Moerke N.J. et al. (2007) Small-molecule inhibition of the interaction between the translation initiation factors eIF4E and eIF4G. Cell 128, 257-267

45. Descamps G. et al. (2012) The cap-translation inhibitor 4EGI1 induces apoptosis in multiple myeloma through Noxa induction. British Journal of Cancer 106, 1660-1667

46. Tamburini J. et al. (2009) Protein synthesis is resistant to rapamycin and constitutes a promising therapeutic target in acute myeloid leukemia. Blood 114, 1618-1627

47. Cencic R. et al. (2011) Reversing chemoresistance by small molecule inhibition of the translation initiation complex eIF4F. Proceedings of the National Academy of Sciences of the United States of America 108, 1046-1051

48. Ko S.Y., Guo H., Barengo N. and Naora H. (2009) Inhibition of ovarian cancer growth by a tumor-targeting peptide that binds eukaryotic translation initiation factor 4E. Clinical Cancer Research 15, 4336-4347

49. Yang H. et al. (2012) In vivo study of breast carcinoma radiosensitization by targeting eIF4E. Biochemical and Biophysical Research Communications 423, 878-883

50. Herbert T.P., Fahraeus R., Prescott A., Lane D.P. and Proud C.G. (2000) Rapid induction of apoptosis mediated by peptides that bind initiation factor eIF4E. Current Biology 10, 793-796

51. Luan F.L. et al. (2003) Rapamycin is an effective inhibitor of human renal cancer metastasis. Kidney International 63, 917-926

52. Seufferlein T. and Rozengurt E. (1996) Rapamycin inhibits constitutive p70s6k phosphorylation, cell proliferation, and colony formation in small cell lung cancer cells. Cancer Research 56, 3895-3897

53. Grewe M., Gansauge F., Schmid R.M., Adler G. and Seufferlein T. (1999) Regulation of cell growth and cyclin D1 expression by the constitutively active FRAP-p70s6 K pathway in human pancreatic cancer cells. Cancer Research 59, 3581-3587

54. Guertin D.A. and Sabatini D.M. (2009) The pharmacology of mTOR inhibition. Science Signaling 2, pe24

55. Rao R.D. et al. (2005) Disruption of parallel and converging signaling pathways contributes to the synergistic antitumor effects of simultaneous mTOR and EGFR inhibition in GBM cells. Neoplasia 7, 921-929

56. Shi Y. et al. (1995) Rapamycin enhances apoptosis and increases sensitivity to cisplatin in vitro. Cancer Research 55, 1982-1988

57. deGraffenried L.A. et al. (2004) Inhibition of mTOR activity restores tamoxifen response in breast cancer cells with aberrant Akt Activity. Clinical Cancer Research 10, 8059-8067
58. Yu K. et al. (2001) mTOR, a novel target in breast cancer: the effect of CCI-779, an mTOR inhibitor, in preclinical models of breast cancer. Endocrine-related Cancer 8, 249-258

59. Del Bufalo D. et al. (2006) Antiangiogenic potential of the Mammalian target of rapamycin inhibitor temsirolimus. Cancer Research 66, 5549-5554

60. Nathan C.O. et al. (2007) Mammalian target of rapamycin inhibitors as possible adjuvant therapy for microscopic residual disease in head and neck squamous cell cancer. Cancer Research 67, 2160-2168

61. Neshat M.S. et al. (2001) Enhanced sensitivity of PTEN-deficient tumors to inhibition of FRAP/mTOR. Proceedings of the National Academy of Sciences of the United States of America 98, 10314-10319

62. Hudes G. et al. (2007) Temsirolimus, interferon alfa, or both for advanced renal-cell carcinoma. The New England Journal of Medicine 356, 2271-2281

63. Witzig T.E. et al. (2005) Phase II trial of single-agent temsirolimus (CCI-779) for relapsed mantle cell lymphoma. Journal of Clinical Oncology 23, 5347-5356

64. Wu C. et al. (2005) Overcoming cisplatin resistance by mTOR inhibitor in lung cancer. Molecular Cancer 4, 25

65. Ito D. et al. (2006) In vivo antitumor effect of the mTOR inhibitor CCI-779 and gemcitabine in xenograft models of human pancreatic cancer. International Journal of Cancer. Journal International du Cancer 118, 2337-2343

66. Wanner K. et al. (2006) Mammalian target of rapamycin inhibition induces cell cycle arrest in diffuse large B cell lymphoma (DLBCL) cells and sensitises DLBCL cells to rituximab. British Journal of Haematology 134, 475-484

67. Rizzieri D.A. et al. (2008) A phase 2 clinical trial of deforolimus (AP23573, MK-8669), a novel mammalian target of rapamycin inhibitor, in patients with relapsed or refractory hematologic malignancies. Clinical Cancer Research 14, 2756-2762

68. Feldman M.E. et al. (2009) Active-site inhibitors of mTOR target rapamycin-resistant outputs of mTORC1 and mTORC2. PLoS Biology 7, e38

69. Thoreen C.C. et al. (2009) An ATP-competitive mammalian target of rapamycin inhibitor reveals rapamycin-resistant functions of mTORC1. Journal of Biological Chemistry 284, 8023-8032

70. Chresta C.M. et al. (2010) AZD8055 is a potent, selective, and orally bioavailable ATP-competitive mammalian target of rapamycin kinase inhibitor with in vitro and in vivo antitumor activity. Cancer Research 70, 288-298

71. Zhao L. et al. (2014) mTOR inhibitor AZD8055 inhibits proliferation and induces apoptosis in laryngeal carcinoma. International Journal of Clinical and Experimantal Medicine 7, 337-347

72. Holt S.V. et al. (2012) Enhanced apoptosis and tumor growth suppression elicited by combination of MEK (selumetinib) and mTOR kinase inhibitors (AZD8055). Cancer Research 72, 1804-1813

73. Yu K. et al. (2010) Beyond rapalog therapy: preclinical pharmacology and antitumor activity of WYE-125132, an ATP-competitive and specific inhibitor of mTORC1 and mTORC2. Cancer Research 70, 621-631

74. Garcia-Martinez J.M. et al. (2009) Ku-0063794 is a specific inhibitor of the mammalian target of rapamycin (mTOR). The Biochemical Journal 421, 29-42

75. Xue Q. et al. (2008) Palomid 529, a novel small-molecule drug, is a TORC1/TORC2 inhibitor that reduces tumor growth, tumor angiogenesis, and vascular permeability. Cancer Research 68, 9551-9557

76. Yu K. et al. (2009) Biochemical, cellular, and in vivo activity of novel ATP-competitive and selective inhibitors of the mammalian target of rapamycin. Cancer Research 69, 6232-6240

77. Garlich J.R. et al. (2008) A vascular targeted pan phosphoinositide 3-kinase inhibitor prodrug, SF1126, with antitumor and antiangiogenic activity. Cancer Research 68, 206-215

78. Maira S.M. et al. (2008) Identification and characterization of NVP-BEZ235, a new orally available dual phosphatidylinositol 3-kinase/mammalian target of rapamycin inhibitor with potent in vivo antitumor activity. Molecular Cancer Therapeutics 7, 1851-1863

79. Schnell C.R. et al. (2008) Effects of the dual phosphatidylinositol 3-kinase/mammalian target of rapamycin inhibitor NVP- 
BEZ235 on the tumor vasculature: implications for clinical imaging. Cancer Research 68, 6598-6607

80. Zou Z.Q. et al. (2009) A novel dual PI3Kalpha/mTOR inhibitor PI-103 with high antitumor activity in non-small cell lung cancer cells. International Journal of Molecular Medicine 24, 97-101

81. Heffron T.P. et al. (2010) Identification of GNE-477, a potent and efficacious dual PI3 K/mTOR inhibitor. Bioorganic \& Medicinal Chemistry Letters 20, 2408-2411

82. Tschopp C. et al. (2000) Phosphorylation of eIF-4E on Ser 209 in response to mitogenic and inflammatory stimuli is faithfully detected by specific antibodies. Molecular Cell Biology Research Communications 3, 205-211

83. Buxade M., Parra-Palau J.L. and Proud C.G. (2008) The Mnks: MAP kinase-interacting kinases (MAP kinase signalintegrating kinases). Frontiers in Bioscience 13, 5359-5373

84. Knauf U., Tschopp C. and Gram H. (2001) Negative regulation of protein translation by mitogen-activated protein kinase-interacting kinases 1 and 2. Molecular and Cellular Biology 21, 5500-5511

85. Wheater M.J., Johnson P.W. and Blaydes J.P. (2010) The role of MNK proteins and eIF4E phosphorylation in breast cancer cell proliferation and survival. Cancer Biology \& Therapy 10, 728-735

86. Lim S. et al. (2013) Targeting of the MNK-eIF4E axis in blast crisis chronic myeloid leukemia inhibits leukemia stem cell function. Proceedings of the National Academy of Sciences of the United States of America 110, E2298-2307

87. Zhang M. et al. (2008) Inhibition of polysome assembly enhances imatinib activity against chronic myelogenous leukemia and overcomes imatinib resistance. Molecular and Cellular Biology 28, 6496-6509

88. Adesso L. et al. (2013) Gemcitabine triggers a pro-survival response in pancreatic cancer cells through activation of the MNK2/eIF4E pathway. Oncogene 32, 2848-2857

89. Sussman A. et al. (2004) Discovery of cercosporamide, a known antifungal natural product, as a selective Pkc1 kinase inhibitor through high-throughput screening. Eukaryotic Cell 3, 932-943

90. Altman J.K. et al. (2013) Inhibition of Mnk kinase activity by cercosporamide and suppressive effects on acute myeloid leukemia precursors. Blood 121, 3675-3681

91. Konicek B.W. et al. (2011) Therapeutic inhibition of MAP kinase interacting kinase blocks eukaryotic initiation factor $4 \mathrm{E}$ phosphorylation and suppresses outgrowth of experimental lung metastases. Cancer Research 71, 1849-1857

92. Cencic R. et al. (2009) Antitumor activity and mechanism of action of the cyclopenta[b]benzofuran, silvestrol. PloS ONE 4, e5223

93. Kim S. et al. (2007) Silvestrol, a potential anticancer rocaglate derivative from Aglaia foveolata, induces apoptosis in LNCaP cells through the mitochondrial/apoptosome pathway without activation of executioner caspase-3 or -7 . Anticancer Research 27(4B), 2175-2183

94. Bordeleau M.E. et al. (2006) RNA-mediated sequestration of the RNA helicase eIF4A by Pateamine A inhibits translation initiation. Chemistry \& Biology 13, 1287-1295

95. Palakurthi S.S. et al. (2000) Inhibition of translation initiation mediates the anticancer effect of the n-3 polyunsaturated fatty acid eicosapentaenoic acid. Cancer Research 60, 29192925

96. Aktas H. et al. (1998) Depletion of intracellular $\mathrm{Ca}^{2+}$ stores, phosphorylation of eIF2alpha, and sustained inhibition of translation initiation mediate the anticancer effects of clotrimazole. Proceedings of the National Academy of Sciences of the United States of America 95, 8280-8285

97. Aktas B.H. et al. (2013) Small-molecule targeting of translation initiation for cancer therapy. Oncotarget 4, 1606-1617

98. Ito T., Warnken S.P. and May W.S. (1999) Protein synthesis inhibition by flavonoids: roles of eukaryotic initiation factor 2alpha kinases. Biochemical and Biophysical Research Communications 265, 589-594

99. Ruggero D. et al. (2004) The translation factor eIF-4E promotes tumor formation and cooperates with c-Myc in lymphomagenesis. Nature Medicine 10, 484-486

100. Choi C.H. et al. (2011) Direct inhibition of eIF4E reduced cell growth in endometrial adenocarcinoma. Journal of Cancer Research and Clinical Oncology 137, 463-469
101. Oridate N., Kim H.J., Xu X. and Lotan R. (2005) Growth inhibition of head and neck squamous carcinoma cells by small interfering RNAs targeting eIF4E or cyclin D1 alone or combined with cisplatin. Cancer Biology \& Therapy 4, 318-323

102. Graff J.R. et al. (2009) eIF4E activation is commonly elevated in advanced human prostate cancers and significantly related to reduced patient survival. Cancer Research 69, 3866-3873

103. Jacobson B.A. et al. (2013) Targeting eukaryotic translation in mesothelioma cells with an eIF4E-specific antisense oligonucleotide. PloS ONE 8, e81669

104. Hayman T.J. et al. (2012) Translation initiation factor eIF4E is a target for tumor cell radiosensitization. Cancer Research $\mathbf{7 2 ,}$ 2362-2372

105. Weiss B., Davidkova G. and Zhou L.W. (1999) Antisense RNA gene therapy for studying and modulating biological processes. Cellular and Molecular Life Sciences 55, 334-358

106. Rinker-Schaeffer C.W., Graff J.R., De Benedetti A., Zimmer S.G. and Rhoads R.E. (1993) Decreasing the level of translation initiation factor $4 \mathrm{E}$ with antisense RNA causes reversal of ras-mediated transformation and tumorigenesis of cloned rat embryo fibroblasts. International Journal of Cancer. Journal International du Cancer 55, 841-847

107. DeFatta R.J., Nathan C.O. and De Benedetti A. (2000) Antisense RNA to eIF4E suppresses oncogenic properties of a head and neck squamous cell carcinoma cell line. The Laryngoscope 110, 928-933

108. Dias N. and Stein C.A. (2002) Antisense oligonucleotides: basic concepts and mechanisms. Molecular Cancer Therapeutics 1, 347-355

109. Dean N.M. and Bennett C.F. (2003) Antisense oligonucleotide-based therapeutics for cancer. Oncogene 22, 9087-9096

110. Mani S. et al. (2002) Phase I clinical and pharmacokinetic study of protein kinase C-alpha antisense oligonucleotide ISIS 3521 administered in combination with 5-fluorouracil and leucovorin in patients with advanced cancer. Clinical Cancer Research 8, 1042-1048

111. Zhang H. et al. (2000) Reduction of liver Fas expression by an antisense oligonucleotide protects mice from fulminant hepatitis. Nature Biotechnology 18, 862-867

112. Yu R.Z. et al. (2004) Tissue disposition of 2'-O-(2-methoxy) ethyl modified antisense oligonucleotides in monkeys. Journal of Pharmaceutical Sciences 93, 48-59

113. Picardi A. et al. (2004) The role of ribavirin in the combination therapy of hepatitis C virus infection. Current Pharmaceutical Design 10, 2081-2092

114. Yan Y., Svitkin Y., Lee J.M., Bisaillon M. and Pelletier J. (2005) Ribavirin is not a functional mimic of the 7-methyl guanosine mRNA cap. RNA 11, 1238-1244

115. Westman B. et al. (2005) The antiviral drug ribavirin does not mimic the 7-methylguanosine moiety of the mRNA cap structure in vitro. RNA 11, 1505-1513

116. Kentsis A. et al. (2005) Further evidence that ribavirin interacts with eIF4E. RNA 11, 1762-1766

117. Grudzien E. et al. (2004) Novel cap analogs for in vitro synthesis of mRNAs with high translational efficiency. RNA $\mathbf{1 0}$ 1479-1487

118. Chen X. et al. (2012) Structure-guided design, synthesis, and evaluation of guanine-derived inhibitors of the eIF4E mRNAcap interaction. Journal of Medicinal Chemistry 55, 38373851

119. Wagner C.R., Iyer V.V. and McIntee E.J. (2000) Pronucleotides: toward the in vivo delivery of antiviral and anticancer nucleotides. Medicinal Research Reviews 20, 417-451

120. Rawal R.K. et al. (2013) 2'-Fluoro-6'-methylene-carbocyclic adenosine phosphoramidate (FMCAP) prodrug: in vitro antiHBV activity against the lamivudine-entecavir resistant triple mutant and its mechanism of action. Bioorganic \& Medicinal Chemistry Letters 23, 503-506

121. Vernachio J.H. et al. (2011) INX-08189, a phosphoramidate prodrug of 6-O-methyl-2'-C-methyl guanosine, is a potent inhibitor of hepatitis $\mathrm{C}$ virus replication with excellent pharmacokinetic and pharmacodynamic properties. Antimicrobial Agents and Chemotherapy 55, 1843-1851

122. Marcotrigiano J., Gingras A.C., Sonenberg N. and Burley S.K. (1999) Cap-dependent translation initiation in eukaryotes is 
regulated by a molecular mimic of eIF4G. Molecular Cell 3, $707-716$

123. Takrouri K. et al. (2014) Structure-activity relationship study of 4EGI-1, small molecule eIF4E/eIF4 G protein-protein interaction inhibitors. European Journal of Medicinal Chemistry 77, 361-377

124. Yefidoff-Freedman R. et al. (2014) 3-substituted indazoles as configurationally locked 4EGI-1 mimetics and inhibitors of the eIF4E/eIF4 G interaction. Chembiochem 15, 595-611

125. Mahalingam P. et al. (2014) Synthesis of rigidified eIF4E/ eIF4 G inhibitor-1 (4EGI-1) mimetic and their in vitro characterization as inhibitors of protein-protein interaction. Journal of Medicinal Chemistry 57, 5094-5111

126. Fan S., Li Y., Yue P., Khuri F.R. and Sun S.Y. (2010) The eIF4E/eIF4 G interaction inhibitor 4EGI-1 augments TRAIL-mediated apoptosis through c-FLIP Down-regulation and DR5 induction independent of inhibition of cap-dependent protein translation. Neoplasia 12, 346-356

127. Panner A., Parsa A.T. and Pieper R.O. (2006) Translational regulation of TRAIL sensitivity. Cell Cycle 5, 147-150

128. Cao J. et al. (2014) Cap-dependent translation initiation factor, eIF4E, is the target for Ouabain-mediated inhibition of HIF1alpha. Biochemical Pharmacology 89, 20-30

129. Peffley D.M., Sharma C., Hentosh P. and Buechler R.D (2007) Perillyl alcohol and genistein differentially regulate $\mathrm{PKB} / \mathrm{Akt}$ and 4E-BP1 phosphorylation as well as eIF4E/ eIF4 $\mathrm{G}$ interactions in human tumor cells. Archives of Biochemistry and Biophysics 465, 266-273

130. Moschetta M., Reale A., Marasco C., Vacca A. and Carratu M.R. (2014) Therapeutic targeting of the mTOR-signalling pathway in cancer: benefits and limitations. British Journal of Pharmacology 171, 3801-3813

131. Eynott P.R. et al. (2003) Effects of cyclosporin A and a rapamycin derivative (SAR943) on chronic allergic inflammation in sensitized rats. Immunology 109, 461-467

132. Karyekar C.S. et al. (2005) A phase I multiple-dose escalation study characterizing pharmacokinetics and safety of ABT-578 in healthy subjects. Journal of Clinical Pharmacology 45, 910-918

133. Efeyan A. and Sabatini D.M. (2010) mTOR and cancer: many loops in one pathway. Current Opinion in Cell Biology 22, $169-176$

134. O'Reilly K.E. et al. (2006) mTOR inhibition induces upstream receptor tyrosine kinase signaling and activates Akt. Cancer Research 66, 1500-1508

135. Guertin D.A. et al. (2009) mTOR complex 2 is required for the development of prostate cancer induced by Pten loss in mice. Cancer Cell 15, 148-159

136. Zoncu R., Efeyan A. and Sabatini D.M. (2011) mTOR: from growth signal integration to cancer, diabetes and ageing. Nature Reviews. Molecular Cell Biology 12, 21-35

137. Harris T.E. and Lawrence J.C. Jr. (2003) TOR signaling. Science's STKE 2003, re15

138. Hara K. et al. (2002) Raptor, a binding partner of target of rapamycin (TOR), mediates TOR action. Cell 110, 177-189

139. Oshiro N. et al. (2004) Dissociation of raptor from mTOR is a mechanism of rapamycin-induced inhibition of mTOR function. Genes to Cells: Devoted to Molecular \& Cellular Mechanisms 9, 359-366
140. Kim D.H. et al. (2002) mTOR interacts with raptor to form a nutrient-sensitive complex that signals to the cell growth machinery. Cell 110, 163-175

141. Wendel H.G. et al. (2007) Dissecting eIF4E action in tumorigenesis. Genes \& Development 21, 3232-3237

142. Topisirovic I., Ruiz-Gutierrez M. and Borden K.L. (2004) Phosphorylation of the eukaryotic translation initiation factor eIF4E contributes to its transformation and mRNA transport activities. Cancer Research 64, 8639-8642

143. Furic L. et al. (2010) eIF4E phosphorylation promotes tumorigenesis and is associated with prostate cancer progression. Proceedings of the National Academy of Sciences of the United States of America 107, 14134-14139

144. Li Y. et al. (2010) Protein phosphatase 2A negatively regulates eukaryotic initiation factor 4E phosphorylation and eIF4F assembly through direct dephosphorylation of Mnk and eIF4E. Neoplasia 12, 848-855

145. Shi Y. et al. (2013) MNK kinases facilitate c-myc IRES activity in rapamycin-treated multiple myeloma cells. Oncogene 32, 190-197

146. Wang X. et al. (2007) Inhibition of mammalian target of rapamycin induces phosphatidylinositol 3-kinase-dependent and Mnk-mediated eukaryotic translation initiation factor 4E phosphorylation. Molecular and Cellular Biology 27, 7405-7413

147. Marzec M. et al. (2011) Simultaneous inhibition of mTORcontaining complex 1 (mTORC1) and MNK induces apoptosis of cutaneous T-cell lymphoma (CTCL) cells. PLoS ONE 6, e24849

148. Bianchini A. et al. (2008) Phosphorylation of eIF4E by MNKs supports protein synthesis, cell cycle progression and proliferation in prostate cancer cells. Carcinogenesis 29, 2279-2288

149. Grzmil M. et al. (2014) MNK1 pathway activity maintains protein synthesis in rapalog-treated gliomas. Journal of Clinical Investigation 124, 742-754

150. Ramalingam S. et al. (2014) First MNKs degrading agents block phosphorylation of eIF4E, induce apoptosis, inhibit cell growth, migration and invasion in triple negative and Her2-overexpressing breast cancer cell lines. Oncotarget 5, 530-543

151. Diab S. et al. (2014) Discovery of 5-(2-(phenylamino)pyrimidin-4-yl)thiazol-2(3H)-one derivatives as potent Mnk2 inhibitors: synthesis, SAR analysis and biological evaluation. ChemMedChem 9, 962-972

152. Jansen A.P., Camalier C.E., Stark C. and Colburn N.H. (2004) 189 Characterization of programmed cell death 4 in multiple human cancers reveals a novel enhancer of drug sensitivity. Molecular Cancer Therapeutics 3, 103-110

153. Kimball S.R. (1999) Eukaryotic initiation factor eIF2. The International Journal of Biochemistry \& Cell Biology 31, 25-29

154. Jagus R., Joshi B. and Barber G.N. (1999) PKR, apoptosis and cancer. The International Journal of Biochemistry \& Cell Biology 31, 123-138

*Corresponding author:

Yafei Cai,

College of Life Sciences,

Anhui Normal University,

Key Laboratory of Biotic Environment and Ecological Safety in

Anhui Province,

Wuhu 241000, Anhui, China.

E-mail: caiyafeinau@163.com 